\title{
Transmission Power Adjustment Scheme for Mobile Beacon-Assisted Sensor Localization
}

\author{
Javad Rezazadeh, Member, IEEE, Marjan Moradi, Member, IEEE, \\ Kumbesan Sandrasegaran, Member, IEEE, Reza Farahbakhsh, Member, IEEE
}

\begin{abstract}
Localization, as a crucial service for sensor networks, is an energy-demanding process for both indoor and outdoor scenarios. GPSbased localization schemes are infeasible in remote, indoor areas and it is not a cost-effective solution for large-scale networks. Single mobile-beacon architecture is recently considered to localize sensor networks with the aim of removing numerous GPS-equipped nodes. The critical issue for the mobile beacon-assisted localization is to preserve the consumed power to increase the lifetime. This paper presents a novel power control scheme, namely "Z-power", for mobile beacon traveling along a predefined path. The proposed scheme takes the advantage of deterministic path traveled by the single beacon to efficiently adjust the transmission power. Based on the extensive results, the proposed power control scheme could successfully improve the beacon and sensors energy consumption about $\mathbf{2 5 . 3 7 \%}$ and $\mathbf{3 4 . 0 9 \%}$, respectively. A significant energy-accuracy trade off was achieved using $Z$-power which could successfully keep the same level of accuracy while providing lower energy consumption. Another group of results collected when obstacle-handling algorithm was applied at the presence of obstacles. In this scenario, Z-power improves energy consumption and localization accuracy with the same level of success.
\end{abstract}

Index Terms-Localization, mobile beacon, path planning, power adjustment, WSN

\section{INTRODUCTION}

W IRELESS Sensor Network (WSN) is a promising technology and one of the most important elements in the Internet of Things (IoT) to provide enormous applications in health, environment monitoring, transport systems, and other commercial areas. Localization techniques are of essential importance to sensor networks because the reported event is meaningful and can be responded to only if its position is known [1]. Localization algorithms for WSN suffer from different challenges and limitations especially at the presence of mobility [2]. Apart from the location precision, the critical challenge is how to reduce the energy consumption and extend the network lifetime long enough to fulfill the deployment objectives [3]. Since the sensors are battery-powered devices with limited resource, the survivability of WSNs depends on power control and power management. Several approaches and schemes have been proposed for dealing with energy consumption in localization. The most effective is exploiting a single mobile beacon to assist positioning and prolong the network lifetime. In this respect, the mobile beacon travels over the region of interest and transmits the beacon signal that includes its location information. Comparatively, mobile beacon-assisted localization is inherently more accurate and costeffective than using static beacons [4, 5]. Despite significant practical benefits offered by these algorithms, a fundamental problem is arisen which is finding an optimum path for mobile beacon trajectory. Our

Javad Rezazadeh, is with the Faculty of Engineering and Information Technology, University of Technology Sydney, Australia and Tehran North Branch, Islamic Azad University, Tehran, Iran. rezazadeh@ieee.org

Marjan Moradi is with the Data61 (NICTA), CSIRO and University of New South Wales, Sydney, Australia.m.moradi@unsw.edu.au

Kumbesan Sandrasegaran is with Faculty of Engineering and Information Technology, University of Technology Sydney, Sydney, Australia. E-mail: \{Javad.rezazadeh, Kumbesan.Sandrasegaran\}@uts.edu.au

Reza Farahbakhsh is a senior researcher in Telecom Sud-Paris Institut Mines Telecom based in France. reza.farahbakhsh@it-sudparis.eu recent research,[4], presented a novel path planning mechanism called Z-curve to assist localization task, effectively. The proposed path tackled critical localization problems like accuracy and time, but it is not an energy efficient method. Energy conserving for mobile beaconassisted localization is an unattended issue. None of the existing paths could achieve a successful trade off between energy efficiency and localization accuracy. In this paper, we take the advantage of deterministic path to apply a power control scheme for mobile beacon. This approach is motivated by the following observations:

1) Considering the fact that a significant proportion of power is spent for packet transmission [6]. The ability of dynamically adjusting the transmit power is promising for power preserving. Employing a transmission Power Control (TPC) mothod could efficiently affects the performance measures of mobile beaconassisted localization, such as energy consumption and network lifetime [7]. Despite the importance of the problem, TPC methods do not have yet considered and applied by any existing static path planning mechanisms.

2) Predefined trajectories for traveling by the mobile beacon provide the opportunity to determine the area in which could be covered. Precisely adjusting transmit power control affects the ability to deliver a packet to its targeted destination [8]. This feature enables unknown nodes to receive sufficient number of beacon messages (three non-collinear messages) and at the same time, mitigates overhearing that is energy costing. The fact that a node listens to the transmitted packets even if these packets are not addressed to it, is called overhearing[9].

Thus, the main contributions of this paper list as follow:

(i) We develop an efficient transmission power adjustment scheme named $Z$-power to minimize the required power for a reliable transmission of beacon messages by the mobile beacon traveling along a static trajectory mechanism.

(ii) We provide a mathematical definition to theoretically analysis the relation between the distance of transmitter and receiver with the required efficient transmission power of the mobile beacon.

(iii) We present power-adapted obstacle handling trajectory mechanism to tolerate obstacles through the path and handle the blocks in a power efficient manner.

\section{LITERATURE REVIEW}

This section is comprised of three subsections: first, an overview of existing power conservation mechanisms are provided. Next, highlighted existing mobile beacon trajectories for location estimation are discussed. Then, few proposed localization techniques merged with power control policies are explained.

\section{A. Power Conservation Mechanisms}

A large body of research exists for energy efficiency in WSN. A survey on energy conservation in WSN can be found in [3]. At a very general level, the authors identified three main enabling techniques, namely, duty cycling, data-driven approaches, and mobility to reduce power consumption in WSN. Power conservation in 
WSNs could be performed by either hardware controls or software algorithms. It leads to introduce a comprehensive classification of the existing power conservation mechanisms into two main categories: Passive and Active [10]. Turning on/off the transceiver in different operational modes (sleep, idle or active) and thus, saving some amount of consumed energy is known as passive power conservation mechanism. In contrast, active refers to the mechanisms that achieve power conservation by utilizing energy-efficient network protocols. One of the effective ways to conserve the limited energy is to control the power at which the nodes transmit signals [11]. Since the transmission of data consumes the greatest amount of energy, adjusting output power at the efficient transmit power level is very crucial. A comprehensive study has been developed in [8] to address some basic principles on power control.

From the implementation perspective, TPC is classified into three different types [12]: network-level, node-level, and packet-level. In network-level, a specific power level is desired to dynamically adjust the output power of all the nodes through the network. In nodelevel, different nodes may use different power levels. It is used to dynamically change the RF power for all outgoing packets to the same desired level. In contrast, in packet-level, each single outgoing packet has its own specific output power so that different power levels may be designated to two consecutive messages.

\section{B. Mobile Beacon Trajectory}

Localization methods can be represented into four groups: (1) static beacons and static nodes such as the methods proposed in [13, 14], (2) static beacons and mobile nodes such as [15], (3) mobile beacons and static nodes [16, 17], and (4) mobile beacons and mobile nodes like the method in [18].

Mobile beacon assisting to localize static nodes is a promising approach and find its way through various applications. From remote or disaster area monitoring or servicing, symptoms detecting in a desired area e.g., agricultural fields to rescuing services are just a few applications of these emerging localization classification. Localization schemes can take advantage of mobility to improve the accuracy and coverage when they rely on mobile beacon with an optimum trajectory. However, how to find the optimum path for the mobile beacon is a challenge. Some fundamental properties of an optimum beacon path have been explained in [16]. The authors made some observations, such as traveling along a shortest path whilst fully covering the region of interest. Moreover, the beacon should also pass closely to as many unknown nodes as possible and provide at least three non-collinear beacon positions.

SCAN, Double SCAN, and Hilbert space filling curve have proposed in [19]. The accuracy of three trajectories directly depends on the resolution of the trajectory (the distance between two successive beacon positions). All the paths have fully coverage, but SCAN suffers from collinearity (beacon messages as transmitted by the mobile beacon when it moves on a straight line). Thus, Double SCAN traverses the field along both directions at the expense of doubling the distance. In [19] the Hilbert curve has been proposed to tackle both the localization and coverage tasks at once, while the key motivation is to solve the collinearity problem of localization where such a curve makes many turns compared with SCAN. This implies that if the mobile beacon moves on the Hilbert curve, the sensors will have the chance to receive noncollinear beacons and obtain a good estimate for their positions. CIRCLES and S-CURVES were proposed in [20] to reduce the amount of straight lines and mitigate the collinearity. Although they produce the shortest path length amongst the other methods, CIRCLES leaves the four corners, uncovered.

The researchers in [21] presented a Scan-based path planning that enables to handle the obstacles while detours around them and broadcasts the beacon messages with a detour flag. An unknown sensor employs the beacon messages with the detour flag to calculate the virtual beacon position and obtain its own location. Once the mobile beacon moves away from the obstacle, it returns back to the original path and start broadcasting the beacon messages again.

The Mobile Anchor node based on Trilateration (LMAT) path has been proposed in [22]. The path could copes with the collinearity but it is unable to maintain the path through the whole network field. It causes to increase the error on the border of the area. Moreover, the path length traveled by the mobile beacon is long.

The authors in [23] proposed a localization method using mobile anchor node and claimed their method is an energy efficient localization because of using non-collinear beacon messages. The anchor node, however, travelled along with a direct line from initial point toward destination. This straight movement path causes some degree of collinearity and decline localization success. Moreover, in the proposed method, the localized stationary sensor nodes assisted to localize unknown nodes. This approach not only decreases the localization accuracy (due to error accumulation) but also increase the power consumption (because of packet transmission by powerlimited unknown nodes).

All the reviewed trajectories make the beacon movement possible along the static deterministic trajectories without the reference to the actual placement of the unknown nodes. In this regard, a few real time or dynamic path planings were introduced by [24, 25]. The major drawback of real time trajectories is the massive number of messages exchanged and high energy consumption.

\section{TPC and Localization}

To the best of our knowledge, there has been limited works to address TPC approach for localization. A prior power adaptive localization algorithm has been introduced in [26] which is based on the DV-Hop method. It is one of the primary localization algorithms which directly employs a power control protocol to enhance the efficiency of location estimation. The adopted power control mechanism aims to improve the accuracy of the estimated distance between the nodes. It is assumed that each node can transmit packets at two fix power levels, though, causes to higher overhead.

In [12], a particle-filtering-based localization algorithm has proposed which is assisted by multiple transmit-power information. The main contribution of the paper is to incorporate the practical multiple transmit-power information into the process of particle filtering. The multiple power scheduling approach improved the accuracy and power efficiency of the localization scheme.

To conclude, a wide body of research on mobile beacon localization exists, none of them considers adjusting transmission power to improve energy efficiency of localization.

In the following sections, we first briefly review Z-curve which is used as the base-trajectory to apply the proposed power control for energy efficient localization. Next, in section IV, our proposed transmission power control scheme namely $Z$-power will be described as the main contribution of this study.

\section{Problem Statement and Solution OVERVIEW}

Energy consumption is the main concern for all WSN services, especially the target applications of mobile beacon- assisted localization. A practical solution is to design and develop an energy preserving trajectory to travel along by the mobile beacon. As discussed earlier, the cause of wasting power in the trajectories is overhearing. It occurs once the beacon message is transmitted too far and is heard by the nodes which is no longer is required or desired to 
be received. Just blindly transmit a beacon message with a constant power level is the problem of the currently developed deterministic path for mobile beacon localization.

Our recent research done in [4] has proposed Z-curve, which successfully mitigated fundamental localization problems such as coverage, location precision, ineffective position rate, and localization time. The basic curve is depicted in Figure 1 and is said to be level (1) where $l=1$. The network field in level (1) is divided into four sub-square, namely $s q_{k},(k=1, \ldots, 4)$ and their centers are shown by $C_{k}$ while $C_{o}$ is the origin of the basic curve . To derive level$l$, a 2-dimensional field must be divided into $4^{l}$ subsquares. The resolution, $d$, is defined as the side length of each sub-square. The mobile beacon connects the centers of the cells while undertaking the Z-curve. Vertices on the basic Z-curve is replaced by the curve level $(l-1)$, which can be appropriately rotated and/or reflected to form the curve in the higher level.

Recall that three non-collinear beacon messages is a fundamental requirement for localization and the fully localized condition is desired. Hence, the communication range, $R_{c}$, should be set in the way that beacon messages transmitted from every beacon position cover the nodes even the ones placed at the border of the field (e.g., $s_{1}$ in Figure 1). Referring to Figure $1, R_{c} \geq \sqrt{\frac{5}{2}} d$ guarantees the fully covered unknown sensors by a mobile beacon traveling along $Z$-curve. Equation 1 formulates the requirement. The details could be found by [4].

$$
\begin{array}{r}
\left(\operatorname{dist}\left(C_{1}, s_{1}\right)\right)^{2}=\left(\frac{d}{2}\right)^{2}+\left(\frac{3 d}{2}\right)^{2} \\
\operatorname{dist}\left(C_{1}, s_{1}\right)=\sqrt{\frac{5}{2}} d \Rightarrow R_{c} \geq \operatorname{dist}\left(C_{1}, s_{1}\right)
\end{array}
$$

The previously developed trajectory is superior, though no strategy is considered for energy efficiency as a critical metric. This research made a great effort to integrate the concept of power control with deterministic path and dynamically adjusting the transmission power of mobile beacon to assist node localization.

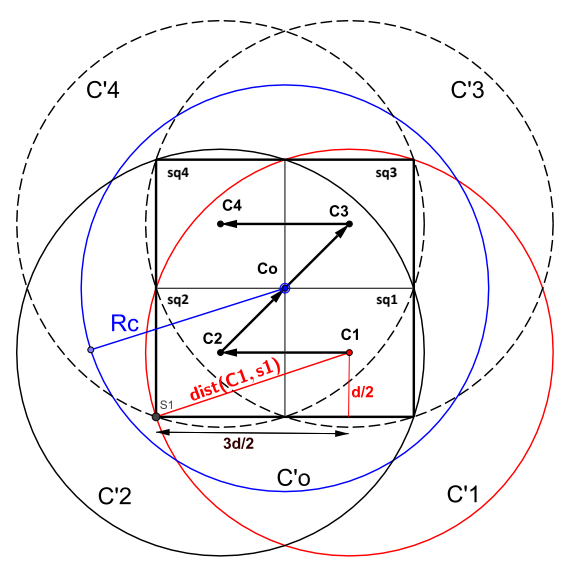

Fig. 1: Transmission radius of beacon positions in basic Z-curve

\section{Z-power: Transmission Power Adjustment Scheme}

The objective of Z-power is to control the transmission power of the mobile beacon to an appropriate level for generating signals in an adaptive manner which are strong enough to reach a specific destination. In this research we examine and formulate the power control scheme for our previously developed path, Z-curve. Though, this study initiates a concrete concept about transmit power control and deterministic path for localization and could be extensible by other designed deterministic paths.
It is desirable to specify the minimum transmission power level that achieves the required communication reliability for the sake of saving power and increasing the system lifetime. To provide system designers with the ability to dynamically control the transmission power, popularly used radio hardware such as CC1000 and CC2420 offers a register to specify the transmission power level during runtime [27].

The proposed Z-power is operated in three different state to assign an efficient power level in each beacon position. Figure 2 illustrates three states in Z-power and the steps are described as follows:

\section{A. Primary State}

Suppose $C_{1} \in s q_{1}$ in Figure 2(a) is the first position traversed for message transmission. In Z-power mechanism, $C_{1}$ is left behind toward $C_{2} \in s q_{2}$ which is the center of adjacent $s q_{2}$ to transmit the second beacon message. These two positions are defined as primary state, $P S$, where the first two messages are received from $C_{1}$ and $C_{2}$. In other words,

$$
P S=\left\{C_{1}, C_{2}\right\}
$$

Let $N_{\left(C_{k}\right)}$ denotes the sensors neighbored with the mobile beacon at position $C_{k}$ either at the same $s q$ or the adjacent $s q$ s. The corresponding $N_{\left(C_{k}\right)}$ of $C_{1}$ and $C_{2}$ is represented by Equation 3:

$$
\begin{aligned}
& N_{\left(C_{1}\right)}=\left\{s_{i} \mid s_{i} \in s q_{1} \cup s q_{2} \cup s q_{3}\right\} \\
& N_{\left(C_{2}\right)}=\left\{s_{i} \mid s_{i} \in s q_{1} \cup s q_{2} \cup s q_{4}\right\}
\end{aligned}
$$

As a result, Equations 2 and 3 define $P S_{r e c}$ as a set of the receiver sensors in the primary state which are supposed to receive the beacon messages from the position. It is mathematically defined by Equation 4.

$$
P S_{r e c}=N_{\left(C_{1}\right)} \cap N_{\left(C_{2}\right)}=\left\{s_{i} \mid s_{i} \in s q_{1} \cup s q_{2}\right\}
$$

It should be noted that the minimum transmission power from positions $C_{1}$ and $C_{2}$ is constrained by $P S_{r e c}$ to achieve the full coverage. Thus, the transmission radius of the mobile beacon in primary state will be set as $R_{c}=\sqrt{\frac{5}{2}} d$ to ensure all unknown nodes resident in $s q_{1}$ and $s q_{2}$ are able to receive both beacon messages from $C_{1}$ and $C_{2}$ positions. The state is illustrated in Figure 2(a).

\section{B. Middle State}

This state enables Z-power to adjust the transmitted power to avoid overhearing and improve the energy efficiency of the mobile beaconassisted localization mechanism. Traveling along the trajectory leads the mobile beacon to meet the center of the main $s q$ which is shown by $C_{o}$.The position is defined as the middle state, $M S$, which is mathematically shown by Equation 5 .

$$
M S=C_{o} \quad \Rightarrow \quad C_{o}=s q_{1} \cap s q_{2} \cap s q_{3} \cap s q_{4}
$$

It is derived from the definition that $N_{\left(C_{o}\right)}$ includes the sensors resident all over the field. In other words:

$$
N_{\left(C_{o}\right)}=\left\{s_{i} \mid s_{i} \in s q_{1} \cup s q_{2} \cup s q_{3} \cup s q_{4}\right\}
$$

Thus, equation 6 leads to specify $M S_{r e c}$, as the set of receiver sensors from middle state that is represented by Equation 7.

$$
M S_{r e c}=N_{\left(C_{o}\right)}=\sum_{i=1}^{n} s_{i}
$$

In order to dynamically adjusting the transmission radius of the mobile beacon in $C_{o}$, the two following constraints must be satisfied to conserve transmission power in $M S$ :

(i) the communication radius must be adjusted in a way that cover the primary state's receivers (obtained from Equation 4). These set of 


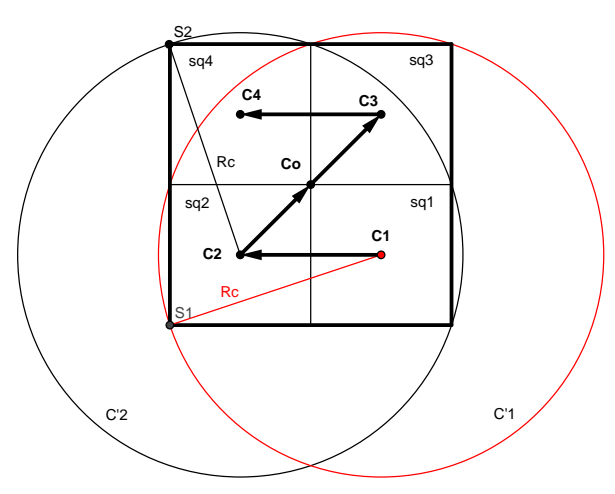

(a) Efficient power for primary-state

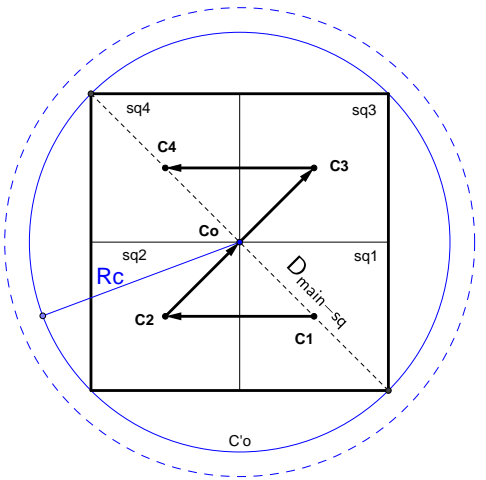

(b) Efficient power for middle-state

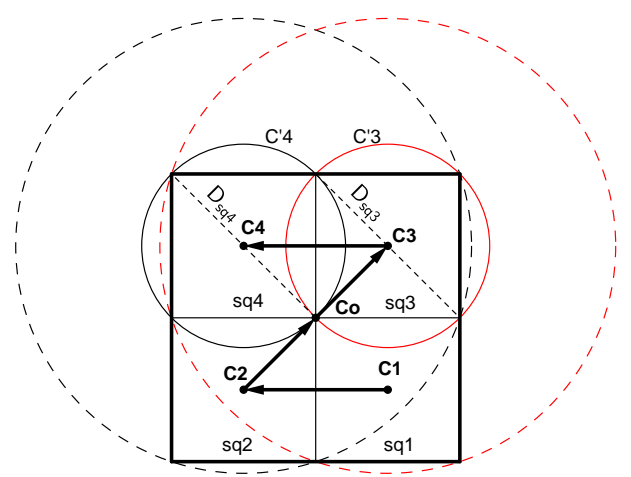

(c) Efficient power for secondary-state

Fig. 2: Efficient power for three states in Z-power

nodes are localizable once they receive the last non-collinear message in middle state.

(ii) The communication radius of the mobile beacon must be adjusted to generate the signal strength just enough for covering the main square, as it is derived from Equation 7.

The constraints are ensured as long as the transmission radius of the state shown by $R_{c(M S)}$ is adjusted based on Equation 8:

$$
\begin{aligned}
D_{(\text {main }-s q)}^{2}=(2 d)^{2}+(2 d)^{2} & \Rightarrow \quad D_{(\text {main }-s q)}=2 \sqrt{2} d \\
R_{c(M S)} & =\frac{1}{2} \times D_{(\text {main }-s q)}=\sqrt{2} d
\end{aligned}
$$

Where the total length of the field is set with $2 d$ and $D_{\text {main-sq }}$ shows the diagonal of the main square. It also is illustrated by Figure 2(b). Consequently, the middle state is desired to draw a circle with the center of $C_{o}$ and the transmission radius of $R_{c(M S)}=\sqrt{2} d$ as encircle of the main square to achieve a reliable transmission. It really goes well without saying that minimizing the communication radius from $\sqrt{\frac{5}{2}} d$ in the original transmission power (dashed line in Figure 2(b)) into $R_{c(M S)}=\sqrt{2} d$ in the middle state tackles the issue of overhearing and consequently, saves the consumed energy.

\section{Secondary State}

Since the mobile beacon obtains the positions $C_{3} \in s q_{3}$ and $C_{4} \in s q_{4}$, the localization task is running to complete in the trajectory. Therefore, $C_{3}$ and $C_{4}$ are defined as secondary state, $S S$ where it helps the mobile beacon to successfully finish the localization task over the trajectory. The unknown sensors located in the $s q_{3}$ and $s q_{4}$ have already received the beacon messages from primary and middle states. Thus, they will be localized once they receive the last non-collinear beacon message. Let $S S_{r e c}$ denotes a set of unknown sensors which should receive the beacon messages from positions $C_{3}$ and $C_{4}$ to be localized which is described by:

$$
S S_{\text {res }}=\left\{s_{i} \mid s_{i} \in s q_{3} \cup s q_{4}\right\}
$$

Based on Equation 9, the last non-collinear beacon message should be received from $C_{3}$ and $C_{4}$ by the corresponding $s_{i}$ which are located at the same $s q$. Consequently, the required transmission range is limited to the radius of the encircle centered at $C_{3}$ for $s_{i} \in s q_{3}$ and $C_{4}$ for $s_{i} \in s q_{4}$. It is derived by Equation 10 .

$$
\begin{aligned}
D_{\left(s q_{k}\right)}^{2}=d^{2}+d^{2} & \Rightarrow D_{\left(s q_{k}\right)}=\sqrt{2} d \\
R_{c(S S)}= & \frac{1}{2} \times D_{\left(s q_{k}\right)}=\frac{\sqrt{2}}{2} d
\end{aligned}
$$

where $D_{s q_{k}}$ represents the diagonal of the various sub-squares that is $s q_{3}$ and $s q_{4}$ in the secondary state. $R_{c(S S)}$ also denotes the adjusted transmission radius of the secondary state which is calculated by Equation 10. The state is illustrated by Figure 2(c).

As a brief discussion on the proposed power control scheme, it is an efficient method in terms of energy consumption for localization. As discussed by [2], mobile beacon trajectories need turning points to avoid collinearity and achieve localization success. However, the higher the turns, the higher the switching power and energy consumption. With this description, our proposed power control scheme is divided into 3 different states to efficiently adjust the transmit power. The number of the states show the different areas created based on the turning points for the basic curve (in Fig.1). Each area has specific features such as the required transmission power to cover the sensors over. Thus, these number of states is promising to achieve a reasonable trade-off between power efficiency and localization success.

\section{Mathematical Model}

This section presents the derived mathematical model for the required transmission power as a function of the distance based on the states discussed in Section IV.

The study presented by [6] have revealed the existence of three distinct reception regions: connected, transitional, and disconnected. Since packet reception rate $\left(P_{r r}\right), P_{r r} \geq 0.9$, it is interpreted that the transmitted packet is received and the distance is defined as the connected region. The transitional region implies the extent of the distance which is characterized by the unreliable reception rate. The disconnected region is defined by the distance where $P_{r r} \leq 0.1$. Figure 3 depicts the packet reception rate $\left(P_{r r}\right)$ for these regions.

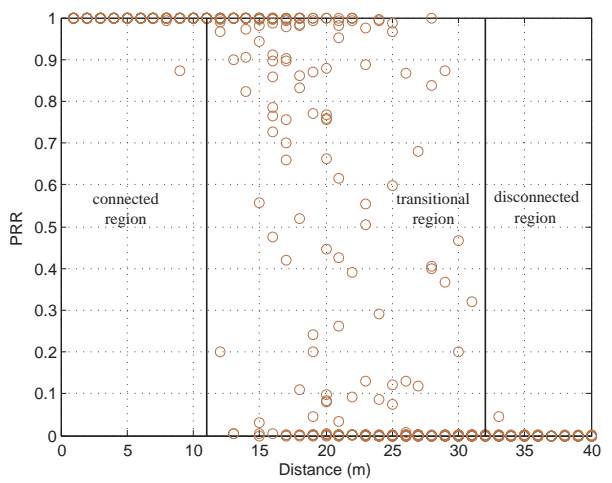

Fig. 3: $P_{r r}$ versus distance. $\left(\sigma=4, P_{L}\left(d_{0}\right)=55 \mathrm{dBm}, \gamma=3.3, P_{\text {trans }}=0 \mathrm{dBm}\right)$

The reception region imposes some restrictions to adjust the transmission power as a function of distance between the mobile beacon transmitter and the unknown sensors receivers. Hence, the transmission power should be adjusted strong enough till the desired 
distances discussed in different states lie within the connected region. The nature of $P_{r r}$ is a Bernoulli random variable which takes value 1 if the packet is received and 0 for failure. It is given by:

$$
P_{r r}=\left(1-P_{b e}\right)^{8 f \times M}
$$

where $f=20$ byte is the size of the frame based on TinyOS [28] after being encoded (the frame consists of preamble, network payload and CRC). Manchester encoding scheme is employed, so $M=2$. $P_{b e}$ is the probability of bit error which depends on the modulation scheme. Here, it selects non-coherent FSK modulation where used in MICA2 motes and formulated by [6]:

$$
P_{b e}=\frac{1}{2} \exp ^{\left(-\frac{S N R}{2} \frac{B_{N}}{R}\right)}
$$

where $B_{N}$ is the noise bandwidth and $R$ is the data rate in bits. MICA2s use the Chipcon CC1000 radio [29] which $R=19.2 \mathrm{kbps}$ and $B_{N}=30 \mathrm{kHz}$. Based on Equations 11, 12 and the values of the variables $R, B_{N}$ and $M$, Equation 13 mathematically represents the $P_{r r}$ based on the applied channel model.

$$
P_{r r}=\left(1-\frac{1}{2} \exp ^{\left(\frac{-S N R}{2} \times \frac{1}{0.64}\right)}\right)^{16 f}
$$

where $S N R$ shows the signal-to-noise ratio. Based on the theoretical models proposed by [6], $P_{r r}$ is given by:

$$
P_{r r}=\left(1-\frac{1}{2} \exp ^{\left(-\frac{10 \frac{S N R^{d B}}{10}}{2} \times \frac{1}{0.64}\right)}\right)^{16 f}
$$

Consider the connected region is bounded to $P_{r r}$ greater than 0.9 . Then, it is obtained from Equation 14 that:

$$
\begin{aligned}
& \left.0.9^{\frac{1}{16 f}}=1-\frac{1}{2} \exp ^{\left(-\frac{10^{\frac{S N R}{10}}}{2}\right.} \times \frac{1}{0.64}\right) \\
& \left.\exp ^{\left(-\frac{10^{\frac{S N R^{d} B}{10}}}{2}\right.} \times \frac{1}{0.64}\right)=2\left(1-0.9^{\frac{1}{16 f}}\right)
\end{aligned}
$$

Based on the mathematical relation between exponential function and natural logarithm, the Equation 16 is represented as:

$$
\begin{aligned}
& 10^{\frac{S N R^{d B}}{10}}=-1.28 \ln \left(2\left(1-0.9^{\frac{1}{16 f}}\right)\right) \\
& \frac{S N R^{d B}}{10}=\log _{10}^{\left(-1.28 \ln \left(2\left(1-0.9^{\frac{1}{16 f}}\right)\right)\right)}
\end{aligned}
$$

Consequently, $S N R$ is denoted by the Equation 19 .

$$
S N R^{d B}=10 \log _{10}^{\left(-1.28 \ln \left(2\left(1-0.9^{\frac{1}{16 f}}\right)\right)\right)}
$$

On the other hand, $S N R$, signal-to-noise ratio is given by:

$$
S N R^{d B}=P_{r e c}^{d B}-P_{n}^{d B}
$$

$P_{\text {rec }}$ shows the reception power and $P_{n}$ defines noise floor. $P_{n}$ depends on both, the radio and the environment [30]. On the other hand, $P_{r e c}$ is:

$$
P_{\text {rec }}^{d B}=P_{\text {trans }}^{d B}-P_{L}^{d B}
$$

By combining Equations 20 and $21, S N R^{d B}$ is defined as:

$$
\begin{aligned}
& S N R^{d B}=P_{\text {trans }}^{d B}-P_{L}(d)^{d B}-P_{n}^{d B} \\
& P_{\text {trans }}^{d B}=S N R^{d B}+P_{L}(d)^{d B}+P_{n}^{d B}
\end{aligned}
$$

Given the $P_{L}(d)^{d B}$ obtained from Equation 23 [30].

$$
P_{L}(d)^{d B}=P_{L}\left(d_{0}\right)^{d B}+10 \gamma \log \left(\frac{d}{d_{0}}\right)+X_{\sigma}^{d B}
$$

where $P_{L}\left(d_{0}\right)$ and $\gamma$ are the power loss at the reference distance $d_{0}$ and the path loss exponent, respectively. Due to the Gaussian characteristic of the log-normal shadowing in the path loss model, $X_{\sigma}=\left(0, \sigma^{2}\right)$ is a Gaussian random variable with mean 0 and standard deviation $\sigma$ (shadowing effect) and $X_{\sigma}$ can be bounded within $\pm 2 \sigma$. Note that shadowing effect is negligible in the cases where $\sigma=0$ which is the condition the node is not tackled with the obstacles. With the aim of obtaining the required transmission power covering the connected region, let $X_{\sigma}$ is set with $+2 \sigma$. So,

$$
P_{\text {trans }}^{d B}=S N R^{d B}+\left(P_{L}\left(d_{0}\right)+10 \gamma \log _{10}^{\left(\frac{d}{d_{0}}\right)}+2 \sigma\right)+P_{n}
$$

Finally, the adjusted transmission power of the proposed mobile beacon assisted localization trajectory at distance $d$ in different states and at the reference distance $d_{0}=1$ is derived by the mathematical model proposed in Equation 25.

$$
P_{\text {trans }}^{d B}=S N R^{d B}+\left(P_{L}\left(d_{0}\right)+10 \gamma \log _{10}^{d}+2 \sigma\right)+P_{n}
$$

To conclude, the derived equation in 25 should be employed to adjust the transmission power in three defined states. In each state, the value $d$ must be plugged by its relevant driven $R_{C}$ to avoid over hearing and conserve the power of mobile beacon. Moreover, applying the driven formula in Equation 25 provides a realistic environment and reflect the radio irregularity condition for packet transmission.

\section{PARAmeter Settings And Evaluation Metrics}

The performance of the proposed power control scheme was evaluated by a series of simulations using MATLAB. We assume a random deployment of static sensors and a single mobile beacon moving around. The beacon has the ability to adjust its transmission power. The critical parameters are listed in Table I. In this research we consider three different methods for position calculation: (1) Weighted Centroid Localization (WCL): Unknown sensors calculate their own positions based on averaging the coordinates of all received beacons. To investigate the impact of different received coordinates, a weight function is defined depends on the RSS value of the mobile beacon at different positions. (2) Time-Priority Trilateration (TPT): unknown sensors are localized with employing the three earlier received messages. The main objective of this approach is estimation of the sensors location within the shortest possible time. (3) Accuracy-Priority Trilateration (APT): unknown sensors are localized with employing the three nearest received messages from the mobile beacon. It provides the chance to estimate the location with higher accuracy while the three strongest RSS values are utilized in trilateration. To analyze and evaluate simulation results, some critical metrics need to be investigated such as the energy efficiency, the localization accuracy, ineffective position rate, localization success, and time. The details of all parameter settings are referred to the our recent research $[4,5]$.

TABLE I: Simulation parameters

\begin{tabular}{ll}
\hline Parameter & Value \\
\hline Network Size & $100 \mathrm{~m} \times 100 \mathrm{~m}$ \\
Unknown Sensors Number & 250 \\
Mobile Beacon Number & 1 \\
Beacon Speed $(\mathrm{m} / \mathrm{s})$ & $0.5,1,2,3,4$ \\
$\frac{R_{c}}{d}$ & $\frac{1}{2}, \frac{3}{4}, 1, \frac{5}{4}, \frac{3}{2}, \frac{7}{4}, 2, \frac{5}{2}, 3$ \\
Path Loss $(\gamma)$ & 3.3 \\
Standard Deviation $(\sigma)$ & $2,4,6,8$ \\
$P_{L}\left(d_{0}\right)$ & $55 \mathrm{~dB}$ \\
$d_{0}$ & 1 \\
Transmission Power $\left(P_{t}\right)$ & $-20 \mathrm{dBm}<P_{t}<10 \mathrm{dBm}$ \\
Simulation Run & 50 \\
\hline
\end{tabular}




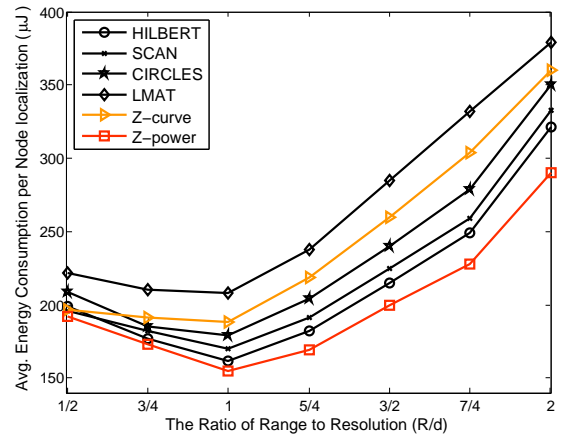

(a) Energy consumption of the mobile beacon

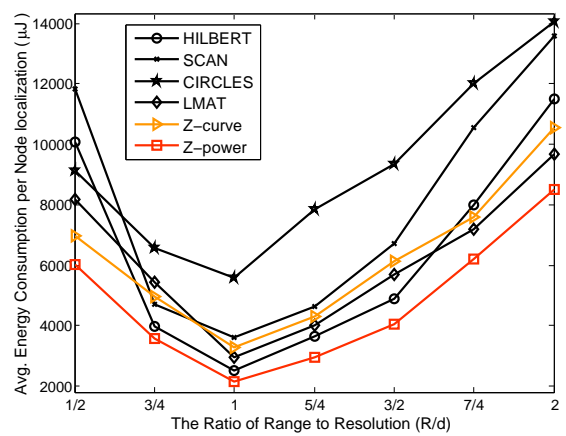

(b) Energy consumption of the unknown sensors

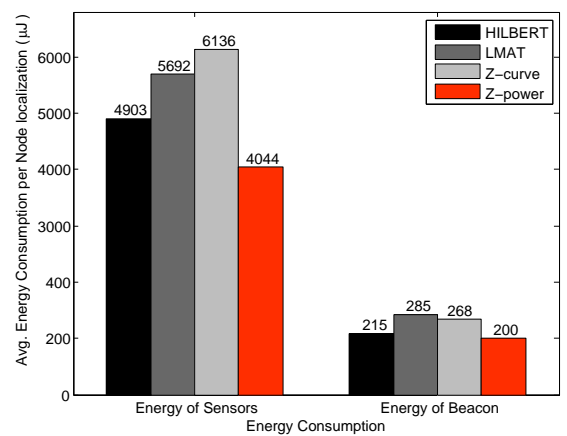

(c) Both sensors and beacon

Fig. 4: Energy consumption comparison

\section{A. Energy Consumption Comparison}

Energy efficiency of both the mobile beacon and unknown sensors is critical to increase the network lifetime. Figure 4 measures the impact of the ratio of $R_{c} / d$ on the consumed energy per node localization by the mobile beacon (Figure 4(a)) and unknown sensors (Figure 4(b)) for various static trajectories. As shown in Figure 4(a), the mobile beacon energy consumption per node localization of all mechanisms have a common feature. The average energy consumption increases with enlarging the ratio of $R_{c} / d$. The required transmission power will be increased for larger communication ranges. The figure also represents that the energy spent per node localization in Z-power is lower than other methods. On the other hand, the impact of the ratio of $R_{c} / d$ on the average energy consumption by the unknown sensors is illustrated by Figure 4(b). In case of the proposed trajectory, the energy consumption is initially more efficient. An important observation is that energy consumption increases with enlarging $R_{c} / d$. In case the $R_{c}=d$, the energy consumption of all trajectories reaches its lowest value.

In figure 4(c), the scheme is compared with the three existing benchmark paths, Z-curve, Hilbert and LMAT in terms of the consumed power for localization by unknown sensors beside the spent energy by the mobile beacon. Although, the mobile beacon is less energy constraint node than the unknown sensors, the resourceconstrained WSN desire to reduce the required power of both nodes. It is obtained from the figure that the proposed Z-power achieves more efficient energy consumption for both nodes.

$Z$-power is more energy efficient than Z-curve, the base-trajectory with no power control. In mobile beacon, energy is conserved with efficient transmit power. In sensor sides, the number of receivers are bounded to the necessary receivers which have been investigated by different states in Section IV.

\section{B. Impact of Z-Power on Localization Accuracy}

The localization precision calculated by APT localization technique is shown by Figure 5(a). The result is reasonable since the three beacon messages employed for localization by APT is selected based on higher RSSI. Hence, in the case of APT the accuracy of the Zpower remains unchanged without considering the transmit power level.

Figure 5(b) illustrates the error estimated by TPT localization method. Z-power improves the accuracy, although the Z-curve trajectory has already been have higher precision than LMAT and Hilbert algorithms. In case of Z-curve, the transmitted beacon position is obtained by some farther unknown sensors with lower signal strength which makes higher localization error. While the range in Z-power is delimiting by the power adjustment scheme, the accuracy increases.

Figure 5(c) investigates the impact of Z-power on error measured by WCL technique. Z-power remarkably degrades the localization error compared with other two trajectories. The achievement is reasonable because more constraints will be placed on the quantity of the received beacon information. The beacon messages contributed in localization have higher signal strength and promise higher accuracy. For more evaluation of Z-power, the average consumed energy by unknown sensors for localization is compared with the obtained localization accuracy estimated in APT technique for $R_{c} / d=3 / 2$ and reports by Figure 5(d). The trade off by the adaptive transmission power scheme is significant. Indeed, Z-power is minimizing the average consumed energy per node localization and improving the location accuracy compared with the other benchmark trajectories.

\section{Comparison of Localization Success and Energy}

Impact of Z-power on localization success, ineffective positions and time is shown in Figure 6. Success in Z-power is compared by Figure 6(a) with the other three trajectories against the average energy consumption per node localization. As observed from the figure, $Z$-power saves significant energy than Z-curve for achieving the same level of success. However, Z-curve obtained $100 \%$ localization success but consumed higher energy per node location estimation than LMAT to successfully localize all 250 unknown sensors.

\section{Comparison of Localization Success and collinearity}

The efficiency of a trajectory for mobile beacon will be confirmed by fewer ineffective positions for higher localization success. In fact, a lower ineffective position rate indicates the smaller number of collinear positions generated by the mobile beacon. As observed from Figure 6(b), Z-power has the smallest number of ineffective position rate compared with $Z$-curve, Hilbert and LMAT. The achievement implies that ineffective position rate is directly affected by the number of received beacons. In other word, the limited transmission radius, the less the number of collinear positions and as a result, the lower ineffective positions.

\section{E. Impact of Z-power on Localization Time}

Figure 6(c) depicts the impact of Z-power on the average required time for localization calculated by APT technique beside TPT. With the power adaptive scheme, the average time slightly decreases for APT method. However, the required localization time is very little affected with the scheme in TPT. The first three received beacon messages are contributed in location estimation by TPT, no matter what size of transmission radius is justified. Z-power shows its effectiveness in APT technique since the three contributed messages for localization will be chosen at the end. In other words, the required localization message selection process must be deferred till the last receivable beacon message will be obtained by the unknown node. Therefore, delimiting the transmission radius has direct impact on shortening the waiting time for localization. 


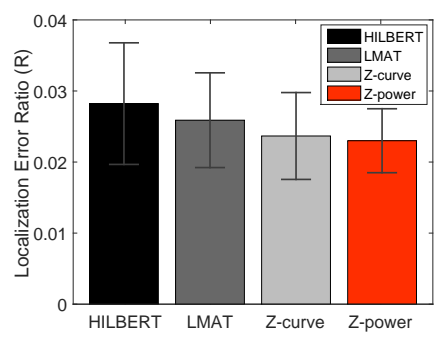

(a) Accuracy in APT technique

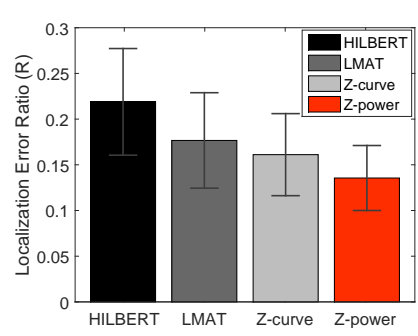

(b) Accuracy in TPT technique

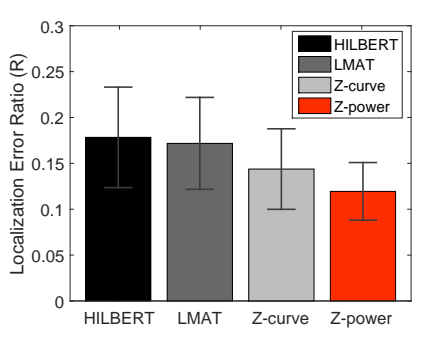

(c) Accuracy in WCL technique

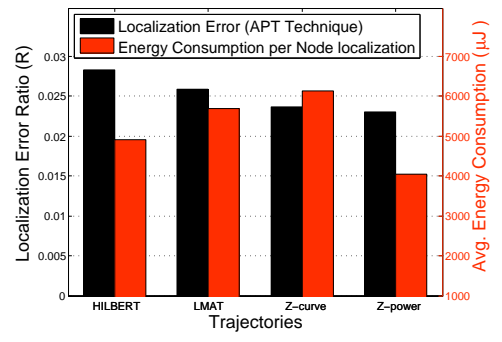

(d) Localization error versus energy

Fig. 5: Localization Accuracy comparison

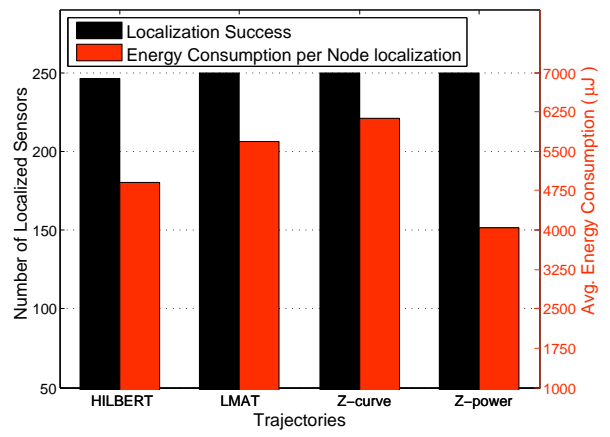

(a) success versus energy consumption

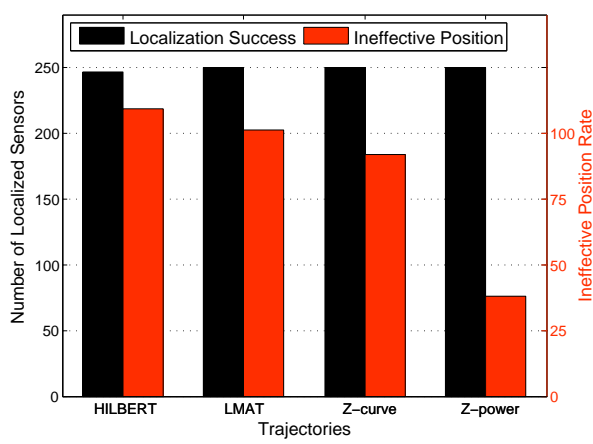

(b) success versus ineffective positions

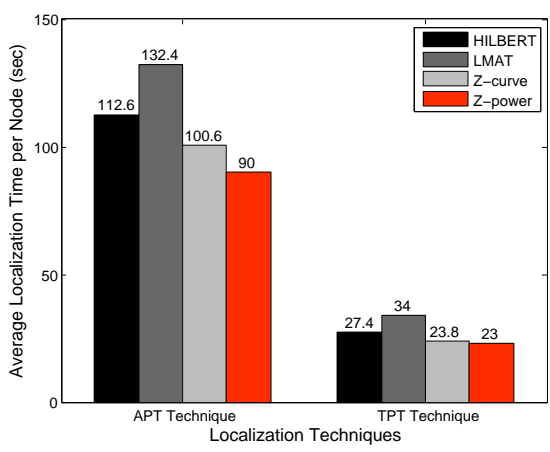

(c) Localization time comparison

Fig. 6: Impact of Z-power on Success, ineffective positions and time

\section{POWER-AdAPted Z-POWER OBSTACle-HANDLING}

The effectiveness of the static trajectory degrades at the presence of obstacles in terms of localization success and accuracy. Even though, in the existing literatures the problem is often overlooked by unrealistic assumption but the mobile beacon traveling along the trajectory may be challenged at the presence of the obstacles as shown in Figure 7. In [4], an obstacle tolerance method named Z-curve obstacle-handling trajectory was presented to handle the obstacles obstructing the path. Here, Z-power obstacle handling trajectory is presented in Section VII-A and the results are reported in Section VII-B. Two different simulation scenarios are considered, either without power control or adapting the power transmission scheme.

As investigated in Section IV, the transmission power adjustment scheme Z-power has been adapted for power conservation. The efficiency motivates the basic idea of this section, obstacle-handling trajectory, how to integrate the power control for better improvement. To deal with the issue, Z-power obstacle-handling trajectory must make a decision of which power is required to adjust for message transmission.

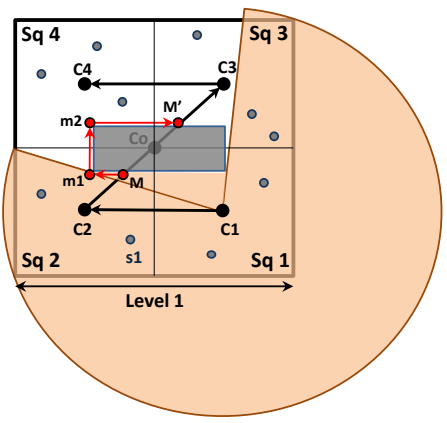

Fig. 7: Z-power obstacle-presence in level(1)

\section{A. Z-power Obstacle-Handling Trajectory}

The algorithm comprises three various phases: obstacle-detection phase, detouring phase, and path resumption phase. The obstacle detection leads the mobile beacon to operate in two different modes, normal-mode or obstacle-mode. In the obstacle-mode, the mobile beacon adopts $Z$-power trajectory in which it moves clockwise around the obstacle and helps in localization. As soon as the mobile beacon changes its movement direction, the beacon positions are broadcasted per each turn. Two sets of messages, changing mode and changing direction, provide the opportunity for unknown sensors to receive sufficient numbers of messages for localization. Once the mobile beacon moves away from the obstacle, the Z-power obstacle-handling trajectory must be terminated and the operational mode will be changed into normal one. Figure 7 depicts this phases, schematically. In order to integrate the proposed power adaptive scheme with the obstacle handling trajectory, Z-power must make a decision of which power is required to adjust for message transmission. On the other hand, a transmitted message for handling the obstacles fall into two different types, either detecting or detouring message. These two type of positions are unknown in the sense of transmission power and need to be further investigated.

Let $C_{k}^{b e h i n d}$ denotes the latter beacon position which has been left behind before the obstacle detection. So, transmission power for both detouring and detecting messages would be assigned according to the power level of $C_{k}^{b e h i n d}$. Once the mobile beacon encounters the obstacle, $N\left(C_{k}^{\text {behind }}\right)$ it can be treated as a set of neighbored sensors of position $C_{k}^{b e h i n d}$ which are uncovered by the transmitted message. Consequently, by applying the proposed power-adapted obstaclehandling trajectory, the unknown sensors of obstacle-presence scenario are localized not only successfully but also power-efficiently.

As an example, $\mathbf{M}, \mathbf{M}^{\prime}, m 1$ and $m 2$ are the messages transmitted around the obstacle as shown in Figure 7. Hence, based on Z-power obstacle handling, the transmitted power must be adjusted to be $R_{c}=$ $\sqrt{\frac{5}{2}} d$ since $C_{k}^{b e h i n d}=C_{2}$ is supported by the primary state.

\section{B. Z-power Obstacle-Handling Results}

This section elaborates a comprehensive simulation results related with Z-power obstacle-handling, as shown in Figure 8. This set of 


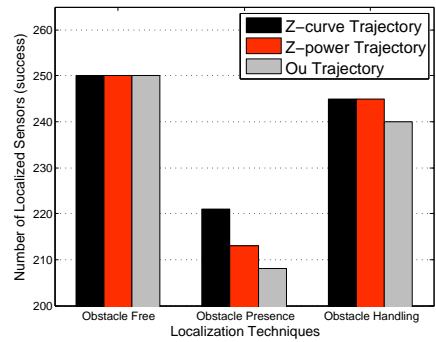

(a) obstacle and success

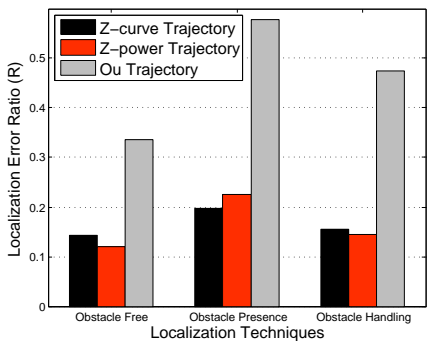

(b) obstacle and accuracy (TPT)

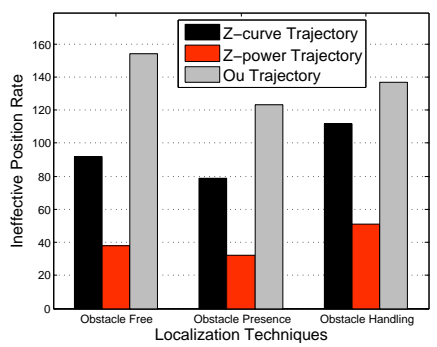

(c) obstacle and collinearity

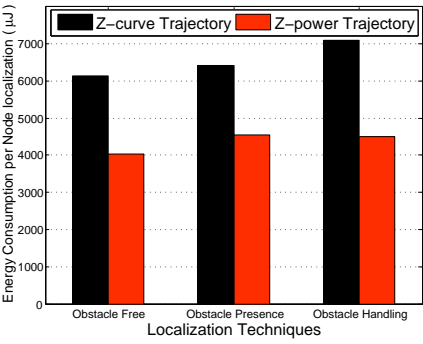

(d) obstacle and energy

Fig. 8: Impact of Z-power obstacle-handling on success, accuracy, collinearity and energy

findings consider the effectiveness and efficiency of the integrated obstacle-handling mechanism with power adjustment scheme. The results are compared with the obstacle-resistance Ou trajectory proposed by [21] and Z-curve [4] to confirm the superiority of Z-power obstacle-handling. The performance is evaluated in terms of energy efficiency, localization success, accuracy and collinearity as follows.

1) Localization Success: As observed in Figure 8(a), Z-power achieves $100 \%$ localization success similar to Z-curve and Ou Scanbased method when the monitoring field is devoid of obstacles. It can also be seen that encountering the obstacles decreases the localization success of Z-curve, Z-power and the Scan-based trajectory for around $11.6 \%, 14.8 \%$ and $16.8 \%$, respectively. By applying obstacle-handling scheme for three methods, the localization success improves at the presence of obstacles. Z-curve trajectory and Z-power obstacle-handling trajectory obtained $98 \%$ localization success and both outperforms Ou method with $96 \%$ localization success.

2) Localization Accuracy: Because of the obstacles, sensor nodes may not obtain accurate beacon messages for estimating their positions. It causes to the higher localization error for different trajectories in obstacle-presence scenario compared with the obstaclefree, as shown in Figure 8(b). It is obtained from the figure that $Z$ power obstacle-handling achieved higher precision than Ou obstaclehandling and Z-curve obstacle-handling. The discrepancy between the developed trajectory and method proposed by [21] is affected by the collinear beacon positions generated by Scan movement pattern. Delimiting the communication range in Z-power leads to receive messages from closer beacon position with higher signal strength and thus lower localization error.

3) Ineffective Position Rate: It should be noted that collinear beacon positions decrease the localization accuracy and energy efficiency. Figure 8(c) plots the efficiency of Z-power obstacle-handling in terms of ineffective position rate. When the mobile beacon encounters the obstacles in the field, collinear positions may degrade because of less number of received messages for three different trajectories. By applying the obstacle-handling rules and transmitting some handling messages around the obstacles, the number of received messages and consequently the number of collinear positions increase. It can be obtained from the figure that ineffective position rate increases about $28 \%$ by Z-power obstacle-handling compared with Z-power in obstacle-free scenario. Aside from that, Ou trajectory can successfully improve ineffective position rate compared with Scan-based trajectory examined in obstacle-free environment. The transmitted messages by detouring flag leads Ou obstacle-handling trajectory to decrease the number of collinear positions and thus, lower ineffective position rate. The detouring flag messages would not be employed by the sensors to estimate the locations.

4) Energy Consumption: Figure 8(d) shows that how Z-power is more energy efficient than Z-curve mechanism. Moreover, dynamically adjusting the transmission power improves the energy efficiency of the Z-power in obstacle-free and obstacle-presence scenarios. On the other hand, encountering the obstacles causes higher energy consumption for both Z-curve and Z-power trajectories. It is a justifiable behaviour since the number of successfully localized sensors has decreased. It should be noted that the energy consumption must be calculated considering the number of successfully localized sensors. The summarized values of the number of successfully localized sensors are shown in Table II. Considering the table, in obstaclepresence scenario, however, no message is transmitted for obstaclehandling the performance of Z-power has quit similar with obstaclehandling scenario. It is due to the lower number of successfully localized sensors in obstacle-presence scenario.

TABLE II: Localization success vs average energy consumption

\begin{tabular}{|c|c|c|c|c|}
\hline \multirow{2}{*}{ Scenario } & \multicolumn{2}{|c|}{ Z-curve } & \multicolumn{2}{c|}{ Z-power } \\
\cline { 2 - 5 } & Success & Avg energy & Success & Avg energy \\
\hline Obst-Free & 250 & 6136 & 250 & 4044 \\
\hline Obst-Presence & 221 & 6411 & 213 & 4541 \\
\hline Obst-Handling & 245 & 7066 & 245 & 4496 \\
\hline
\end{tabular}

\section{CONCLUSION}

The ability of dynamically adjusting transmission power can potentially save energy in localization, and thus, enlarges the network life time. In this paper, energy efficiency for mobile beacon-assisted localization was considered. We developed a power-adaptive transmission scheme, Z-power, to adjust an efficient power level for a reliable transmission. Extensive results confirmed the efficiency of the proposed scheme for mobile-beacon assisted localization which traversed a deterministic path. The proposed power control scheme could successfully improve the beacon and sensors energy consumption about $25.37 \%$ and $34.09 \%$, respectively. Moreover, a poweradjustment scheme for a non-idealistic environment was developed to handle the obstacles in a real environment. The results reported that our developed power-adjustment scheme could successfully localize a significant numbers of sensors (245 out of 250 nodes) in obstaclehandling scenario at the expense of a negligible amount of energy (less than $10 \%$ increment). As future direction of this research, we are planning to evaluation of the presented idea on a multiple Z-curve areas as well as on a real dataset can be considered as future work.

\section{REFERENCES}

[1] Z. Chen, Q. Zhu, and Y. C. Soh, "Smartphone inertial sensor-based indoor localization and tracking with ibeacon corrections," IEEE Transactions on Industrial Informatics, vol. 12, no. 4, Aug 2016.

[2] S. Halder and A. Ghosal, "A survey on mobility-assisted localization techniques in wireless sensor networks," J. Netw. Comput. Appl., vol. 60, no. C, pp. 82-94, Jan. 2016.

[3] G. Anastasi, M. Conti, M. D. Francesco, and A. Passarella, "Energy conservation in wireless sensor networks: A survey." Ad Hoc Networks, vol. 7, pp. 537-568, 2009.

[4] J. Rezazadeh, M. Moradi, A. S. Ismail, and E. Dutkiewicz, "Superior path planning mechanism for mobile beacon-assisted localization in wireless sensor networks," Sensors Journal, IEEE, vol. 14, 2014. 
[5] J. rezazadeh, M. Moradi, A. S. Ismail, and E. Dutkiewicz, "Impact of static trajectories on localization in wireless sensor networks," Wirel. Netw., vol. 21, no. 3, pp. 809-827, Apr. 2015.

[6] M. Z. n. Zamalloa and B. Krishnamachari, "An analysis of unreliability and asymmetry in low-power wireless links," ACM Transactions on Sensor Networks, vol. 3, 2007.

[7] H. Cotuk, K. Bicakci, B. Tavli, and E. Uzun, "The impact of transmission power control strategies on lifetime of wireless sensor networks," IEEE Trans. Comput., vol. 63, no. 11, Nov. 2014.

[8] V. Kawadia and P. Kumar, "Principles and protocols for power control in wireless ad hoc networks," Selected Areas in Communications, IEEE Journal on, vol. 23, no. 1, Jan 2005.

[9] W. Ye, J. Heidemann, and D. Estrin, "An energy-efficient mac protocol for wireless sensor networks," in INFOCOM, IEEE, 2002.

[10] N. A. Pantazis and D. D. Vergados, "A survey on power control issues in wireless sensor networks," IEEE Communications Surveys Tutorials, vol. 9, no. 4, pp. 86-107, 2007.

[11] S. Sengupta, M. Chatterjee, and K. Kwiat, "A game theoretic framework for power control in wireless sensor networks," IEEE Transactions on Computers, vol. 59, no. 2, Feb 2010.

[12] H. Ren and M. Q. H. Meng, "Power adaptive localization algorithm for wireless sensor networks using particle filter," IEEE Transactions on Vehicular Technology, vol. 58, no. 5, pp. 2498-2508, Jun 2009.

[13] A. E. Assaf, S. Zaidi, S. Affes, and N. Kandil, "Low-cost localization for multihop heterogeneous wireless sensor networks," IEEE Transactions on Wireless Communications, vol. 15, no. 1, pp. 472 484, Jan 2016.

[14] T. Bui, P. Xu, N. Phan, W. Zhu, and G. Wu, "An accurate and energy-efficient localization algorithm for wireless sensor networks," in 2016 IEEE 83rd Vehicular Technology Conference (VTC Spring), May 2016, pp. 1-5.

[15] J. Rezazadeh, M. Moradi, and A. Ismail, "Efficient localization via middle-node cooperation in wireless sensor networks," in Electrical, Control and Computer Engineering (INECCE), 2011 International Conference on, 2011, pp. 410-415.

[16] G. Han, J. Jiang, C. Zhang, T. Q. Duong, M. Guizani, and G. K. Karagiannidis, "A survey on mobile anchor node assisted localization in wireless sensor networks," IEEE Communications Surveys Tutorials, vol. 18, no. 3, pp. 2220-2243, thirdquarter 2016.

[17] G. Han, X. Yang, L. Liu, M. Guizani, and W. Zhang, "A disaster management-oriented path planning for mobile anchor node-based localization in wireless sensor networks," IEEE Transactions on Emerging Topics in Computing, vol. PP, no. 99, pp. 1-1, 2017.

[18] S. Hartung, A. Kellner, K. Rieck, and D. Hogrefe, "Monte carlo localization for path-based mobility in mobile wireless sensor networks," in 2016 IEEE Wireless Communications and Networking Conference, April 2016, pp. 1-7.

[19] D. Koutsonikolas, S. M. Das, and Y. C. Hu, "Path planning of mobile landmarks for localization in wireless sensor networks," Computter Communication, vol. 30, 2007.

[20] R. Huang and G. Zaruba, "Static path planning for mobile beacons to localize sensor networks," in Pervasive Computing and Communications Workshops, 2007. PerCom, 2007.

[21] C. Ou and W. He, "Path planning algorithm for mobile anchor based localization in wireless sensor networks," Sensors Journal, IEEE, 2011.

[22] G. Han, H. Xu, J. Jiang, L. Shu, T. Hara, and S. Nishio, "Path planning using a mobile anchor node based on trilateration in wireless sensor networks," Wireless Communications and Mobile Computing, 2011.

[23] B. Zhang and F. Yu, "An energy efficient localization algorithm for wireless sensor networks using a mobile anchor node," in 2008 International Conference on Information and Automation, June 2008, pp. 215-219.

[24] X. Li, N. Mitton, I. Simplot-Ryl, and D. Simplot-Ryl, "Dynamic beacon mobility scheduling for sensor localization," Parallel and Distributed Systems, IEEE Transactions on, vol. 23, 2012.

[25] C.-T. Chang, C.-Y. Chang, and C.-Y. Lin, "Anchor-guiding mechanism for beacon-assisted localization in wireless sensor networks,"
Sensors Journal, IEEE, vol. 12, pp. 1098 -1111, 2012.

[26] W.-H. Liao, K.-P. Shih, and Y.-C. Lee, "A localization protocol with adaptive power control in wireless sensor networks," Comput. Commun., vol. 31, no. 10, pp. 2496-2504, Jun. 2008.

[27] S. Lin, F. Miao, J. Zhang, G. Zhou, L. Gu, T. He, J. A. Stankovic, S. Son, and G. J. Pappas, "Atpc: Adaptive transmission power control for wireless sensor networks," ACM Trans. Sen. Netw. vol. 12 , no. 1, pp. 6:1-6:31, Mar. 2016.

[28] P. Levis and S. Madden, "Tinyos: An operating system for sensor networks," in Ambient Intelligence, W. Weber, J. Rabaey, and E. Aarts, Eds. Springer, 2005, pp. 115-148.

[29] Chipcon. CC1000 low power radio transceiver, http://www.chipcon.com.

[30] T. Rappaport, Wireless Communications: Principles and Practice, 2nd ed. Prentice Hall PTR, 2001.

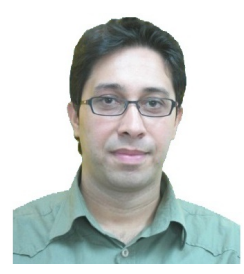

Javad Rezazadeh is a researcher at University of Technology, Sydney (UTS) in Australia and also an assistant professor at the Azad University of North Tehran Branch in Iran. He holds a PhD in Wireless Communications from University Technology Malaysia (UTM) while being received Academic Excellent Student Award for the top 1 postgraduate in the year 2014. His research interests include localization Systems, Internet of Things (IoT) and Mobile Computing.

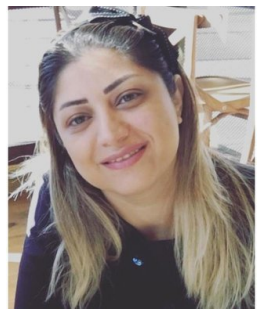

Marjan Moradi is currently pursuing the Ph.D. degree in computer science at the Universiti New South Walse (UNSW) and also researcher at Data61, CSIRO, Australia. She Received the M.Sc. degree by research in computer science from University Technology Malaysia (UTM) while being received Excellent Researcher Award in 2013. Her research interests include mobile networks, Millimeter wave networking, IoT and machine learning.

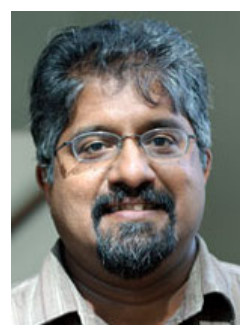

Kumbesan Sandrasegaran is an Associate Professor at UTS. He holds a Ph.D. in Electrical Engineering from McGill University, Canada, 1994. His current research work focuses on two main areas radio resource management in mobile networks, and engineering of remote monitoring systems for novel applications with industry through the use of embedded systems, sensors and communications systems. He has published over 100 refereed publications and 20 consultancy reports spanning telecommunication and computing systems.

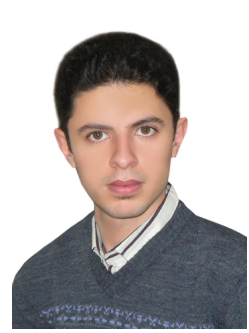

Reza Farahbakhsh is a research fellow at InstitutMines Telecom, Telecom SudParis. He received his $\mathrm{PhD}$ degree from Institut-Mines Telecom, Telecom SudParis (CNRS Lab UMR5157) jointly with Paris VI (UPMC) at 2015. His research interest is Online Social networks, IoT, predictive maintenance, Large scale measurement, User Behavior Analysis. He is actively involved in europeen projects and represent France in two EU Cost actions. 